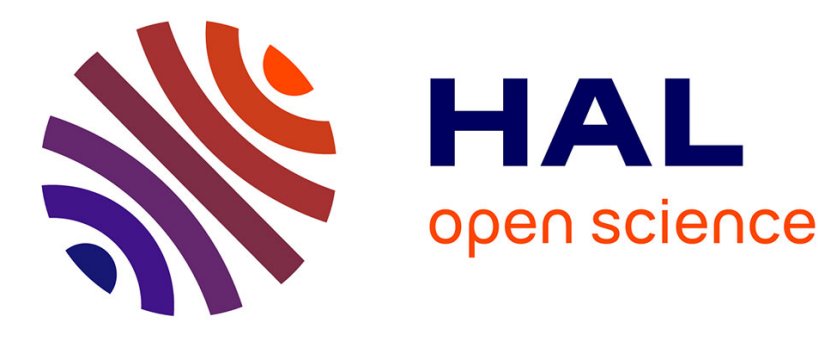

\title{
Fault diagnosis in Takagi-Sugeno nonlinear systems
}

\author{
Dalil Ichalal, Benoît Marx, José Ragot, Didier Maquin
}

\section{To cite this version:}

Dalil Ichalal, Benoît Marx, José Ragot, Didier Maquin. Fault diagnosis in Takagi-Sugeno nonlinear systems. 7th IFAC Symposium on Fault Detection, Supervision and Safety of Technical Processes, Safeprocess 2009, Jun 2009, Barcelone, Spain. pp.CDROM, 10.3182/20090630-4-ES-2003.00084 . hal-00376455

\section{HAL Id: hal-00376455 \\ https://hal.science/hal-00376455}

Submitted on 31 Mar 2014

HAL is a multi-disciplinary open access archive for the deposit and dissemination of scientific research documents, whether they are published or not. The documents may come from teaching and research institutions in France or abroad, or from public or private research centers.
L'archive ouverte pluridisciplinaire HAL, est destinée au dépôt et à la diffusion de documents scientifiques de niveau recherche, publiés ou non, émanant des établissements d'enseignement et de recherche français ou étrangers, des laboratoires publics ou privés. 


\title{
Fault diagnosis for Takagi-Sugeno nonlinear systems
}

\author{
Dalil Ichalal * Benoit Marx* José Ragot * Didier Maquin * \\ * Centre de Recherche en Automatique de Nancy (CRAN), Nancy-Université, \\ CNRS, 2 avenue de la forêt de Haye F-54516 Vandoeuvre-les-Nancy. \\ \{dalil.ichalal, benoit.marx, jose.ragot, didier.maquin\}@ensem.inpl-nancy.fr
}

\begin{abstract}
This paper addresses a new scheme for fault diagnosis in nonlinear systems described by Takagi-Sugeno multiple models. Two cases are considered, the first one concerns the T-S models with known premise variables (the input or the output of the system). For the second case it is supposed that the weighting functions depend on unmeasurable premise variables (state of the system). The approach is based on the design of observer-based residual generator by minimization of the disturbances effect and maximizing the effects of the faults. The synthesis is based on the $\mathcal{L}_{2}$ formalism developed for linear systems. The convergence conditions are given in LMI formulation.
\end{abstract}

Keywords: Fault diagnosis, Nonlinear systems, Takagi-Sugeno systems, LMI formulation.

\section{INTRODUCTION}

Linear models are largely studied and an important literature is devoted to this class of systems. Although they provide solutions for many problems, nonlinear behavior are often present in practical systems then reduce the domain of applicability and the performances of the tools developed for linear models. Indeed, a linear model only represents the behavior of the system around a local operating point. It is known that nonlinear systems are complex and difficult to study, so all the works on the nonlinear systems concern only specific classes, but there is no a general framework like for linear systems. One of the more interesting classes of nonlinear systems is the Takagi-Sugeno (TS) multiple model form which was introduced in Takagi and Sugeno [1985]. It is proved in Tanaka and Wang [2001] that often nonlinear behaviors can be represented exactly or approximated by TS multiple models. The main advantage of these models is the ability to extend the tools designed in the linear system framework. Indeed, many topics of control are extended to TS systems, such as stability and stabilization in Tanaka et al. [1998], Guerra et al. [2006], Chadli et al. [2002], observers and state estimation in Akhenak et al. [2007], Bergsten et al. [2002].

Due to an increasing demand for higher performances, as well as for higher safety and reliability, the model-based approaches to fault diagnosis for dynamic systems have received more attention these last years Patton et al. [1989], Chen and Zhang [1991], Chen et al. [1996], Ding and Frank [1989], Marx et al. [2003]. Concerning the TS fuzzy systems few efforts have been made in fault detection and isolation. Nevertheless we can cite the method based on observers in Akhenak et al. [2007], Marx et al. [2007].

In this paper an observer-based approach is developed for robust residual generator and diagnosis which minimizes the sensitivity to the disturbances and maximizes the sensitivity to the faults. Two cases are studied. The first case concerns the T$\mathrm{S}$ systems with measurable premise variables and the second one deals with the systems with unmeasurable premise variables. The paper is organized as follows, section 2 gives some notations and states the problem. Robust residual generation is tackled in section 3. An LMI-based design of the residual generator is proposed. The proposed observers are used for RFD in section 4 and before concluding, a numerical example is given.

\section{PROBLEM STATEMENT}

Consider the following continuous-time TS nonlinear system subject to faults $f(t)$ and disturbances $d(t)$ given by

$$
\left\{\begin{array}{l}
\dot{x}(t)=\sum_{i=1}^{r} \mu_{i}(\xi(t))\left(A_{i} x(t)+B_{i} u(t)+E_{i} d(t)+F_{i} f(t)\right) \\
y(t)=\sum_{i=1}^{r} \mu_{i}(\xi(t))\left(C_{i} x(t)+D_{i} u(t)+G_{i} d(t)+R_{i} f(t)\right)
\end{array}\right.
$$

where $A_{i} \in R^{n \times n}, B_{i} \in \mathbb{R}^{n \times n_{u}}, C_{i} \in R^{n_{y} \times n}, D_{i} \in R^{n_{y} \times n_{u}}$, $E_{i} \in R^{n \times n_{d}}, F_{i} \in R^{n \times n_{f}}, G_{i} \in R^{n_{y} \times n_{d}}$, and $R_{i} \in R^{n_{y} \times n_{f}}$.

The weighing functions $\mu_{i}$ are nonlinear and depend on the decision variable $\xi(t)$ which can be measurable like $\{u(t), y(t)\}$ or not measurable like the state $x(t)$ of the system. The weighting functions satisfy the following properties:

$$
\left\{\begin{array}{l}
0 \leq \mu_{i}(\xi(t)) \leq 1 \\
\sum_{i=1}^{r} \mu_{i}(\xi(t))=1
\end{array}\right.
$$

Thus the structure of the multiple model is simple and is a universal approximator since it can represent any nonlinear behavior according to an adequate number $r$ of the local models (chap 14 of Tanaka and Wang [2001]). The multiple model structure provides a mean to generalize the tools developed for linear systems to nonlinear systems due to the properties expressed in (2).

The input signals $u(t), f(t)$ and $d(t)$ belong in $\mathcal{L}_{2}$ set. The $\mathcal{L}_{2^{-}}$ norm of $u(t) \in \mathcal{L}_{2}$ is given by $\|u\|_{2}=\sqrt{\int_{0}^{+\infty} u^{T} u d t}$. 
In the field of observer design and diagnosis of nonlinear systems using multiple model approach, Patton et al. [1998] proposed an observer-based method to generate residual generator and using an observer bank in order to achieve isolation, an application to DC motor is proposed. In Akhenak et al. [2007], a sliding mode observer for TS systems is proposed to detect and estimate actuator faults. In these works, the authors assumed that the weighting functions depend on measurable premise variables (input or output) of the system. It is clear that the choice of measurable premise variables offers a good simplicity to generalize the methods already developed for linear systems. But in the case where the premise variables are not measurable, the problem becomes very hard. However, this formalism is very important in both the exact representation of the nonlinear behavior by multiple model (see the simulation example) and in diagnosis method based on observer banks to detect and isolate actuator and/or sensor faults. Indeed in this case, the use of measurable premise variables requires to develop two different multiple models, but using multiple models with unmeasurable premise variables allows to develop only one model of the system behavior to detect and isolate both actuator and sensor faults using observer banks. In the literature, few works are devoted to the case of unmeasurable decision variables. Nevertheless, we can cite Bergsten et al. [2002], Palm and Bergsten [2000], where the authors proposed the fuzzy ThauLuenberger observer which is an extension of the classical Luenberger observer. The main contribution of this paper is to propose a method for fault diagnosis of nonlinear systems described by TS models with measurable and unmeasurable premise variables using the standard $H_{\infty}$ framework developed for linear systems.

\section{RESIDUAL GENERATOR DESIGN}

The residual generator design for nonlinear systems described by Takagi-Sugeno multiple model is addressed in this section. Two cases are studied, the first case deals with TS models where the decision variables are measurable and the second one concerns TS models with unmeasurable decision variables.

\section{1 case 1: measurable premise variables}

Let consider the TS nonlinear system subject to disturbances and sensor and actuator faults modeled in (1) An observerbased residual generator is proposed in the following form

$$
\left\{\begin{array}{l}
\dot{\hat{x}}(t)=\sum_{i=1}^{r} \mu_{i}(\xi)\left(A_{i} \hat{x}(t)+B_{i} u(t)+L_{i}(y(t)-\hat{y}(t))\right) \\
\hat{y}(t)=\sum_{i=1}^{r} \mu_{i}(\xi)\left(C_{i} \hat{x}(t)+D_{i} u(t)\right) \\
r(t)=\stackrel{M}{ }(y(t)-\hat{y}(t))
\end{array}\right.
$$

where $\hat{x}(t) \in R^{n}$ is the estimated state vector and $r(t) \in R^{n_{f}}$ is the residual signal that is structured in order to be sensitive to the fault $f(t)$. The matrices $L_{i} \in R^{n \times n_{y}}$ and $M \in R^{n_{f} \times n_{y}}$ are the residual generator gains. The objective is to design the gains $L_{i}$ and $M$ in order to minimize the transfer from the disturbances $w(t)$ and to maximize the transfer of the faults $f(t)$ to the residual signal $r(t)$. Let define the state estimation error $e(t)=x(t)-\hat{x}(t)$. Its dynamic is deduced from (1) and (3) as follows

$$
\left\{\begin{array}{l}
\dot{e}(t)=A_{\xi} e(t)+E_{\xi} d(t)+F_{\xi} f(t) \\
r(t)=C_{\xi} e(t)+G_{\xi} d(t)+R_{\xi} f(t)
\end{array}\right.
$$

where

$$
\begin{aligned}
A_{\xi} & =\sum_{i=1}^{r} \sum_{k=1}^{r} \mu_{i}(\xi) \mu_{j}(\xi)\left(A_{i}-L_{i} C_{k}\right) \\
E_{\xi} & =\sum_{i=1}^{r} \sum_{k=1}^{r} \mu_{i}(\xi) \mu_{j}(\xi)\left(E_{i}-L_{i} G_{k}\right) \\
F_{\xi} & =\sum_{i=1}^{r} \sum_{k=1}^{r} \mu_{i}(\xi) \mu_{j}(\xi)\left(F_{i}-L_{i} R_{k}\right) \\
C_{\xi} & =\sum_{i=1}^{r} \mu_{i}(\xi) M C_{i}, G_{\xi}=\sum_{i=1}^{r} \mu_{i}(\xi) M G_{i}, \\
R_{\xi} & =\sum_{i=1}^{r} \mu_{i}(\xi) M R_{i}
\end{aligned}
$$

For convenience, the system (4) can be written under the following compact form

$$
r=G_{r d} d+G_{r f} f
$$

where $G_{r d}$ represents the transfer from the disturbances $d(t)$ to $r(t)$ and defined by

$$
G_{r d}:=\left(\begin{array}{c|c}
A_{\xi} & E_{\xi} \\
\hline M C_{i} & G_{\xi}
\end{array}\right)
$$

and $G_{r f}$ is the transfer from $f(t)$ to $r(t)$ which is defined by

$$
G_{r f}=\left(\begin{array}{c|c}
A_{\xi} & F_{\xi} \\
\hline C_{\xi} & R_{\xi}
\end{array}\right)
$$

In standard $H_{\infty}$ framework (see figure 1), the maximization of

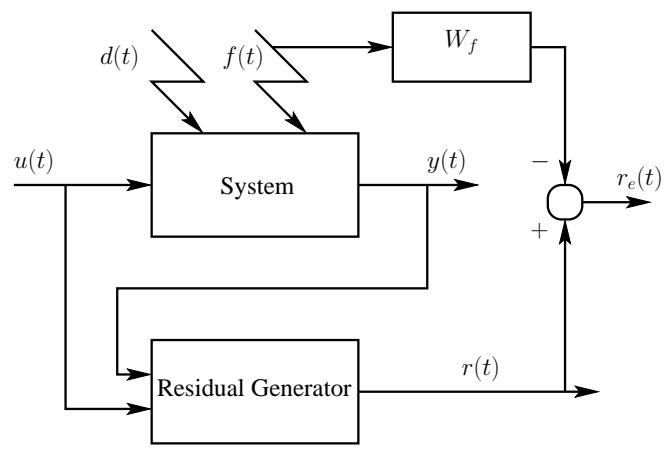

Fig. 1. Scheme of robust residual generation

the effect of the faults $f(t)$ on the residual $r(t)$ can be expressed as a minimization problem. Indeed, by introducing a weighting parameter $W_{f}$, the problem is reduced to a minimization of the effect of the faults on the residual error

$$
r_{e}(t)=r(t)-W_{f} f(t)
$$

As explained in Stoustrup and Niemann [2000] the FDI problem depends on the selected structure of the weight parameter $W_{f}$. Indeed, the fault estimation problem is obtained when $W_{f}=I$ and the detection problem is considered when $W_{f} \in$ $R^{1 \times n_{f}}$. In addition, $W_{f}$ can be chosen as a dynamic parameter. Consider the parameter $W_{f}$ defined

$$
W_{f}=\left(\begin{array}{c|c}
A_{f} & B_{f} \\
\hline C_{f} & D_{f}
\end{array}\right)
$$

$W_{f} \in \mathcal{S}$ where $\mathcal{S}$ is the set of stable filters which have the following property

$$
\left\|W_{f}\right\|_{-}=i n f_{w \in R}\left(\underline{\sigma}\left(W_{f}(j w)\right)\right) \geq 1
$$

(see Mazars et al. [2008] and Mazars et al. [2006] for more details). The interest of this kind of filters is that there is no 
attenuation of the faults but only an amplification on all frequency ranges which improves the problem of fault detection. The detection, isolation and estimation of the faults can be considered by an appropriate choice of the matrices $A_{f}, B_{f}, C_{f}$ and $D_{f}$. The FDI problem is then formulated as the following multi-objective optimization problem

Obtain $L_{i}$ and $M$ which minimize $a \gamma_{f}+(1-a) \gamma_{d}$ where $a \in\left[\begin{array}{ll}0 & 1\end{array}\right]$ subject to the following constraints

$$
\begin{array}{r}
\left\|G_{r f}-W_{f}\right\|_{\infty}<\gamma_{f} \\
\left\|G_{r d}\right\|_{\infty}<\gamma_{d}
\end{array}
$$

System (4) is stable

The theorem 1 gives an LMI method to solve the optimization problem and provides the residual generator gains $L_{i}$ and $M$.

Theorem 1. Given a positive parameter $a \in[0,1]$ and a weighting function $W_{f} \in \mathcal{S}$. The residual generator (3) exists if there exist matrices $P_{1}=P_{1}^{T}>0, P_{2}=P_{2}^{T}>0$ and gain matrices $K_{i}$ and $M$ and positive scalars $\bar{\gamma}_{f}$ and $\bar{\gamma}_{d}$ solution of the following optimization problem

$$
\min _{L_{i}, M, P_{1}, P_{2}, K_{i}, \bar{\gamma}_{f}, \bar{\gamma}_{d}} a \bar{\gamma}_{f}+(1-a) \bar{\gamma}_{d}
$$

s.t.

$$
\begin{gathered}
\left(\begin{array}{cccc}
X_{i k}^{1} & 0 & P_{1} F_{i}-K_{i} R_{k} & C_{k}^{T} M^{T} \\
0 & X_{f}^{2} & P_{2} B_{f} & -C_{f}^{T} \\
F_{i}^{T} P_{1}-R_{k}^{T} K_{i}^{T} & B_{f}^{T} P_{2} & -\bar{\gamma}_{f} I & R_{k}^{T} M^{T}-D_{f}^{T} \\
M C_{k} & -C_{f} & M R_{k}-D_{f} & -I
\end{array}\right)<0 \\
\left(\begin{array}{ccc}
X_{i k}^{1} & P_{1} E_{i}-K_{i} G_{k} & C_{k}^{T} M^{T} \\
E_{i}^{T} P_{1}-G_{k}^{T} K_{i}^{T} & -\bar{\gamma}_{d} I & G_{k}^{T} M^{T} \\
M C_{k} & M G_{k} & -I
\end{array}\right)<0
\end{gathered}
$$

where

$$
\begin{gathered}
X_{i k}^{1}=A_{i}^{T} P_{1}+P_{1} A_{i}-K_{i} C_{k}-C_{k}^{T} K_{i}^{T} \\
X_{f}^{2}=A_{f}^{T} P_{2}+P_{2} A_{f} \\
\forall i, k=1, \ldots, r
\end{gathered}
$$

The gains $L_{i}$ are derived from

$$
L_{i}=P_{1}^{-1} K_{i} \quad i=1, \ldots, r
$$

and the attenuation levels are given by

$$
\gamma_{d}=\sqrt{\overline{\gamma_{d}}} \quad \gamma_{f}=\sqrt{\bar{\gamma}_{f}}
$$

Proof. In faulty case without disturbances the residual generator is reduced to $r=G_{r f} f$. In order to maximize the effects of faults on the residual we consider the weighting stable filter $W_{f}(s)$ defined in (10). Then the maximization problem can be formulated as a minimization problem by solving (12). $G_{r f}-W_{f}$ can be written in the following form

$$
G_{r f}-W_{f}:=\left(\begin{array}{cc|c}
A_{\xi} & 0 & F_{\xi} \\
0 & A_{f} & B_{f} \\
\hline C_{\xi} & -C_{f} & R_{\xi}-D_{f}
\end{array}\right)
$$

Let define a positive and symmetric bloc diagonal matrix

$$
P=\left(\begin{array}{cc}
P_{1} & 0 \\
0 & P_{2}
\end{array}\right)
$$

Using the bounded real lemma Boyd et al. [1994], the condition (12) is formulated as follows

$$
\left(\begin{array}{cccc}
A_{\xi}^{T} P_{1}+P_{1} A_{\xi}^{T} & 0 & P_{1} F_{\xi} & C_{\xi}^{T} \\
0 & A_{f}^{T} P_{2}+P_{2} A_{f}^{T} & P_{2} B_{f} & -C_{f}^{T} \\
F_{\xi}^{T} P_{1} & B_{f}^{T} P_{2} & -\gamma_{f}^{2} I & R_{\xi}^{T}-D_{f}^{T} \\
C_{\xi} & -C_{f} & R_{\xi}-D_{f} & -I
\end{array}\right)<0
$$

Using the definitions (5) of the matrices $A_{\xi}, F_{\xi}, C_{\xi}$ and $R_{\xi}$ and the convex property of the weighing function, the following inequalities are obtained from (23)

$$
\left(\begin{array}{cccc}
X_{i k}^{1} & 0 & P_{1} F_{i}-P_{1} L_{i} R_{k} & C_{k}^{T} M^{T} \\
0 & X_{f}^{2} & P_{2} B_{f} & -C_{f}^{T} \\
F_{i}^{T} P_{1}-R_{k}^{T} K_{i}^{T} & B_{f}^{T} P_{2} & -\gamma_{f}^{2} I & R_{k}^{T} M^{T}-D_{f}^{T} \\
M C_{k} & -C_{f} & M R_{k}-D_{f} & -I
\end{array}\right)<0
$$

where

$$
\begin{gathered}
X_{i k}^{1}=A_{i}^{T} P_{1}+P_{1} A_{i}-P_{1} L_{i} C_{k}-C_{k}^{T} L_{i}^{T} P_{1} \\
X_{f}^{2}=A_{f}^{T} P_{2}+P_{2} A_{f}^{T} \\
i, k=1, \ldots, r
\end{gathered}
$$

In order to obtain the linear matrix inequality (15), we use the change of variables $K_{i}=P_{1} L_{i}$ and $\bar{\gamma}_{f}=\gamma_{f}^{2}$ and $\bar{\gamma}_{d}=\gamma_{d}^{2}$.

In the fault-free case with disturbances, a similar way, by using the bounded real lemma, allows to obtain the LMI (16). The block $(1,1)$ of the the LMI (16) ensures the stability of the observer (i.e. the system (4) is stable) and the robustness against disturbances.

Now, in the faulty case with disturbances, the relative importance of minimizing the effects of the disturbances and maximizing the effects of the faults on the residual signal can be expressed as a minimization of the linear combination $a \gamma_{f}+(1-a) \gamma_{d}$ where $a \in\left[\begin{array}{ll}0 & 1\end{array}\right]$.

\section{2 case 2: unmeasurable premise variables}

In this section, it is assumed that the weighting functions $\mu_{i}$ of the TS nonlinear system (1) depend on the unmeasurable state $x(t)$ of the system. The weighting functions of the residual generator then depend on the estimated state $\hat{x}(t)$ as follows

$$
\left\{\begin{array}{l}
\dot{\hat{x}}(t)=\sum_{i=1}^{r} \mu_{i}(\hat{x})\left(A_{i} \hat{x}(t)+B_{i} u(t)+L_{i}(y(t)-\hat{y}(t))\right) \\
\hat{y}(t)=\sum_{i=1}^{r} \mu_{i}(\hat{x})\left(C_{i} \hat{x}(t)+D_{i} u(t)\right) \\
r(t)=M(y(t)-\hat{y}(t))
\end{array}\right.
$$

By adding and subtracting the term

$$
\sum_{j=1}^{r} \mu_{j}(\hat{x}(t))\left(A_{j} x(t)+B_{j} u(t)\right)
$$

in state equation of the system (1) and the term

$$
\sum_{j=1}^{r} \mu_{j}(\hat{x}(t))\left(C_{j} x(t)+D_{j} u(t)\right)
$$

in the output equation of (1) and by some manipulations using the convex property of the weighting functions (2) the following equivalent system is obtained

$$
\left\{\begin{array}{l}
\dot{x}(t)=\sum_{i=1}^{r} \sum_{j=1}^{r} \mu_{i}(x) \mu_{j}(\hat{x})\left(\tilde{A}_{i j} x(t)+\tilde{B}_{i j} u(t)+E_{i} d(t)+F_{i} f(t)\right) \\
y(t)=\sum_{i=1}^{r} \sum_{j=1}^{r} \mu_{i}(x) \mu_{j}(\hat{x})\left(\tilde{C}_{i j} x(t)+\tilde{D}_{i j} u(t)+G_{i} d(t)+R_{i} f(t)\right)
\end{array}\right.
$$

where

and

$$
\begin{aligned}
& \tilde{A}_{i j}=A_{j}+\Delta A_{i j}, \tilde{C}_{i j}=C_{j}+\Delta C_{i j} \\
& \tilde{B}_{i j}=B_{j}+\Delta B_{i j}, \tilde{D}_{i j}=D_{j}+\Delta D_{i j}
\end{aligned}
$$

$$
\Delta X_{i j}=X_{i}-X_{j}, \quad X_{i} \in\left\{A_{i}, B_{i}, C_{i}, D_{i}\right\}
$$




$$
i, j=1, \ldots, r
$$

After calculating the dynamic of the state estimation error, the following is obtained

$$
\left\{\begin{array}{l}
\dot{e}(t)=\tilde{A}_{x \hat{x}} e(t)+\Delta \tilde{A}_{x \hat{x}} x(t)+\tilde{B}_{x \hat{x}} \tilde{d}(t)+\tilde{F}_{x \hat{x}} f(t) \\
r(t)=\tilde{C}_{x \hat{x}} e(t)+\Delta \tilde{C}_{x \hat{x}} x(t)+\tilde{G}_{x \hat{x}} \tilde{d}(t)+\tilde{R}_{x \hat{x}} f(t)
\end{array}\right.
$$

Using the simplified notation

$$
\sum_{i, j, k=1}^{r} \mu_{i} \hat{\mu}_{j} \hat{\mu}_{k}
$$

instead of

$$
\sum_{i=1}^{r} \sum_{j=1}^{r} \sum_{k=1}^{r} \mu_{i}(x) \mu_{j}(\hat{x}) \mu_{k}(\hat{x})
$$

the matrices of (29) are defined by

$$
\begin{gathered}
\tilde{A}_{x \hat{x}}=\sum_{i, j, k=1}^{r} \mu_{i} \hat{\mu}_{j} \hat{\mu}_{k}\left(A_{j}-L_{j} C_{k}\right) \\
\tilde{B}_{x \hat{x}}=\sum_{i, j, k=1}^{r} \mu_{i} \hat{\mu}_{j} \hat{\mu}_{k}\left[\left(\Delta B_{i j}-L_{j} \Delta D_{i k}\right)\left(E_{i}-L_{j} G_{i}\right)\right] \\
\tilde{F}_{x \hat{x}}=\sum_{i, j, k=1}^{r} \mu_{i} \hat{\mu}_{j} \hat{\mu}_{k}\left(F_{i}-L_{j} R_{i}\right) \\
\tilde{C}_{x \hat{x}}=\sum_{i, j, k=1}^{r} \mu_{i} \hat{\mu}_{j} \hat{\mu}_{k} M C_{k}, \\
\tilde{G}_{x \hat{x}}=\sum_{i, j, k=1}^{r} \mu_{i} \hat{\mu}_{j} \hat{\mu}_{k}\left[M \Delta D_{i k} M G_{i}\right] \\
\tilde{R}_{x \hat{x}}=\sum_{i, j, k=1}^{r} \mu_{i} \hat{\mu}_{j} \hat{\mu}_{k} M R_{i} \\
\Delta \tilde{A}_{x \hat{x}}=\sum_{i, j, k=1}^{r} \mu_{i} \hat{\mu}_{j} \hat{\mu}_{k}\left(\Delta A_{i j}-L_{j} \Delta C_{i k}\right) \\
\Delta \tilde{C}_{x \hat{x}}=\sum_{i, j, k=1}^{r} \mu_{i} \hat{\mu}_{j} \hat{\mu}_{k} M \Delta C_{i k} \\
\tilde{d}(t)=\left[u(t)^{T} d(t)^{T}\right]^{T}
\end{gathered}
$$

Let define the augmented state vector $\tilde{x}=\left[\begin{array}{ll}e^{T} & x^{T}\end{array}\right]^{T}$. Then from (29) The residual vector $r$ is then given by

$$
r=G_{r d} \tilde{d}+G_{r f} f
$$

where

$$
G_{r d}=\left(\begin{array}{cc|c}
\tilde{A}_{x \hat{x}} & \Delta \tilde{A}_{x \hat{x}} & \tilde{B}_{x \hat{x}} \\
0 & A_{x} & \tilde{B}_{x} \\
\hline \tilde{C}_{x \hat{x}} & \Delta \tilde{C}_{x \hat{x}} & \tilde{G}_{x \hat{x}}
\end{array}\right)
$$

and

$$
\begin{gathered}
G_{r f}=\left(\begin{array}{cc|c}
\tilde{A}_{x \hat{x}} \Delta \tilde{A}_{x \hat{x}} & \tilde{F}_{x \hat{x}} \\
0 & A_{x} & F_{x} \\
\hline \tilde{C}_{x \hat{x}} \Delta \tilde{C}_{x \hat{x}} & \tilde{R}_{x \hat{x}}
\end{array}\right) \\
A_{x}=\sum_{i=1}^{r} \mu_{i}(x) A_{i}, F_{x}=\sum_{i=1}^{r} \mu_{i}(x) F_{i} \\
\tilde{B}_{x}=\sum_{i=1}^{r} \mu_{i}(x)\left[B_{i} E_{i}\right]
\end{gathered}
$$

The FDI problem is the same as the problem given in (12)-(14). In order to determine the gains $L_{i}$ and $M$ of the residual generator (27), the theorem 2 gives an LMI solution of the problem (12)-(14) extended to TS nonlinear systems with unmeasurable premise variables.
Theorem 2. Given a positive parameter $a$ and a weighting function $W_{f}$. The residual generator (3) exists if there exist matrices $P_{1}=P_{1}^{T}>0, P_{2}=P_{2}^{T}>0$ and gain matrices $K_{i}$ and $M$ and positive scalars $\bar{\gamma}_{1}$ and $\bar{\gamma}_{2}$ solution of the following optimization problem

$$
\min _{L_{i}, M, P_{1}, P_{2}, K_{i}, \bar{\gamma}_{f}, \bar{\gamma}_{d}} a \bar{\gamma}_{f}+(1-a) \bar{\gamma}_{d}
$$

s.t.

$$
\begin{aligned}
& \left(\begin{array}{ccccc}
X_{j k}^{1} & \Xi_{i j k} & 0 & P_{1} F_{i}-K_{j} R_{i} & C_{k}^{T} M^{T} \\
* & X_{i}^{2} & 0 & P_{2} F_{i} & \Delta C_{i k}^{T} M^{T} \\
* & * & X_{f}^{3} & P_{3} B_{f} & -C_{f}^{T} \\
* & * & * & -\bar{\gamma}_{f} I & \left(M R_{i}-D_{f}\right)^{T} \\
* & * & * & * & -I
\end{array}\right)<0 \\
& \left(\begin{array}{ccccc}
X_{j k}^{1} & \Xi_{i j k} & P_{1} \Delta B_{i j}-K_{j} \Delta D_{i k} & P_{1} E_{i}-K_{j} G_{i} & C_{k}^{T} M^{T} \\
* & X_{i}^{2} & P_{2} B_{i} & P_{2} E_{i} & \Delta C_{i k}^{T} M^{T} \\
* & * & -\bar{\gamma}_{\tilde{d}} I & 0 & \Delta D_{i k}^{T} M^{T} \\
* & * & * & -\bar{\gamma}_{\tilde{d}} I & G_{i}^{T} M^{T} \\
* & * & * & * & -I
\end{array}\right)<0
\end{aligned}
$$

where

$$
\begin{gathered}
X_{j k}^{1}=A_{j}^{T} P_{1}+P_{1} A_{j}-K_{j} C_{k}-C_{k}^{T} K_{j}^{T} \\
X_{i}^{2}=A_{i}^{T} P_{2}+P_{2} A_{i} \\
X_{f}^{3}=A_{f}^{T} P_{3}+P_{3} A_{f} \\
\Xi_{i j k l}=P_{1} \Delta A_{i j}-K_{j} \Delta C_{i k} \\
\forall i, j, k=1, \ldots, r
\end{gathered}
$$

The gains $L_{i}$ are derived from

$$
L_{i}=P_{1}^{-1} K_{i} \quad i=1, \ldots, r
$$

and the attenuation levels are given by

$$
\gamma_{d}=\sqrt{\bar{\gamma}_{d}} \quad \gamma_{f}=\sqrt{\bar{\gamma}_{f}}
$$

Proof. After calculating the augmented system with $\tilde{x}=$ $\left[\begin{array}{lll}e^{T} & x^{T} & x_{f}^{T}\end{array}\right]^{T}$ by including the filter $W_{f}$ which has $x_{f}$ as a state vector and calculating $r_{e}(t)=r(t)-r_{f}(t)$ where $r_{f}(t)$ is the output of the filter $W_{f}$ (see figure 1), the proof follows exactly the steps which have been given for the proof of the theorem 1 .

Remark 1. Note that the theorem 2 is more general that the theorem 1 . Indeed, if the weighting functions $\mu_{i}$ of the system (1) depend on measurable premise variables, the problem given in the theorem 1 can be deduced from the theorem 2 by taking $i=j$. When the premise variable is the state of the system, if $C_{1}=C_{2}=\ldots=C$ and the number of the sub-models $r$ is important, it might be, therefore, difficult to find a common matrix $P$ defined by

$$
P=\left(\begin{array}{ccc}
P_{1} & 0 & 0 \\
0 & P_{2} & 0 \\
0 & 0 & P_{3}
\end{array}\right)
$$

satisfying the conditions of theorem 2 (see remark 6.1 in Tanaka et al. [1998]).

\section{ROBUST FAULT DIAGNOSIS}

Due to the presence of exogenous disturbances, the residual signals are different from zero even in the fault-free case. In the framework of fault detection, a threshold based on the obtained attenuation levels $\gamma_{f}$ and $\gamma_{d}$ is generated. An alarm is generated by comparison between the residual signals $r(t)$ and the threshold. A fixed threshold is determined as follows

$$
J_{t h}=\gamma_{d} \rho
$$


where $\rho$ is the bound of $d(t)$ in the measurable premise variables case and it represent the bound of $\tilde{d}(t)$ in the unmeasurable premise variables. The decision logic is given by

$$
\left\{\begin{array}{l}
\left|r_{i}(t)\right|<J_{t h} \Rightarrow \text { no fault } \\
\left|r_{i}(t)\right|>J_{t h} \Rightarrow \text { fault }
\end{array}\right.
$$

In order to improve the fault detection, a residual generator can be constructed for each fault separately. Each residual generator is designed to minimize the transfer from $f_{i}$ to $r_{e i}=r_{i}$ $W_{f i} f_{i}, i=1, \ldots, n_{f}$.

In the unmeasurable premise variables case, the system is seen as an uncertain system. The input $u(t)$ then appear in the dynamic of state estimation error. The method proposed in this paper considers the input $u(t)$ as a perturbation as $d(t)$ and by considering the new perturbation vector $\tilde{d}(t)=\left[u(t)^{T} d(t)^{T}\right]^{T}$ the problem is solved. It is clear that considering the input $u(t)$ as a perturbation penalizes the fault detection because the computed threshold depends on the upper bound of $\tilde{d}(t)$. Using the method proposed in Casavola et al. [2008] for linear systems with polytopic uncertainties where $u(t)$ is considered as a perturbation to minimize separately from $d(t)$. Indeed, instead of minimizing the index $\left(a \bar{\gamma}_{f}+(1-a) \bar{\gamma}_{\tilde{d}}\right)$ under the LMI constraints, the index which has been used in Casavola et al. [2008] described by $\left(a \bar{\gamma}_{f}+b \bar{\gamma}_{d}+c \bar{\gamma}_{u}\right)$ can be used. An adaptive threshold can be then generated using a timewindowed rms-norm (see Casavola et al. [2008], Frank and Ding [1997]).

It is often considered that the fault vector $f(t)$ has two components, the first one noted $f_{a}(t)$ represent the vector of the faults affecting only the actuator, thus, they appear in the state equation. The second component noted $f_{s}(t)$ is the vector of the faults affecting only the sensors. The output of the system is always given by

$$
y(t)=\sum_{i=1}^{r} \mu_{i}(\xi)\left(C_{i} x(t)+D_{i} u(t)+G_{i} d(t)+R_{i} f(t)\right)
$$

but in the case where the faults $f_{a}(t)$ do not affect the output of the system, the matrices $R_{i}$ are not full rank. As pointed out in (Stoustrup and Niemann [2000]), in this case, where $W_{f}=I$ the attenuation level $\gamma_{f}$ becomes greater than 1 the problems in theorem 1 and 2 does not have a solution. In order to avoid this problem, a perturbation term is added in the output equation as follows

$$
y(t)=\sum_{i=1}^{r} \mu_{i}(\xi)\left(C_{i} x+D_{i} u+G_{i} d+\left[\begin{array}{ll}
\varepsilon_{i} & R_{i}^{1}
\end{array}\right]\left[\begin{array}{l}
f_{a} \\
f_{s}
\end{array}\right]\right)
$$

where $\varepsilon_{i}$ are the matrices of distribution of the actuator faults $f_{a}(t)$ in the output equation and are chosen as small as possible. However, in the context of fault isolation, this approach may generate false alarms. To improve the isolation results, we propose to add and subtract the perturbation term and make the added term to ensure the full rank of $R_{i}$ and consider the subtract term as a perturbation to minimize

$$
y(t)=\sum_{i=1}^{r} \mu_{i}(\xi)\left(C_{i} x+D_{i} u+\bar{G}_{i} \bar{d}+\bar{R}_{i}\left[\begin{array}{l}
f_{a} \\
f_{s}
\end{array}\right]\right)
$$

where

$$
\bar{G}_{i}=\left[\begin{array}{ll}
G_{i} & b \varepsilon_{i}
\end{array}\right], \bar{R}_{i}=\left[\begin{array}{ll}
\varepsilon_{i} & R_{i}^{1}
\end{array}\right], \bar{d}=\left[\begin{array}{c}
d \\
-\frac{f_{a}}{b}
\end{array}\right]
$$

where $b$ is a positive real parameter. Using this second approach, the threshold $J_{t h}$ is calculated by using the bound of the new perturbation vector $\bar{d}(t)$, thus the fault isolation is improved.

\section{NUMERICAL EXAMPLE}

The proposed algorithm of robust diagnosis is illustrated by an academic example. Let consider the nonlinear system (1) defined by

$$
\begin{gathered}
A_{1}=\left[\begin{array}{ccc}
-2 & 1 & 1 \\
1 & -3 & 0 \\
2 & 1 & -8
\end{array}\right], A_{2}=\left[\begin{array}{ccc}
-3 & 2 & -2 \\
5 & -3 & 0 \\
1 & 2 & -4
\end{array}\right], \\
B_{1}=\left[\begin{array}{c}
1 \\
5 \\
0.5
\end{array}\right], B_{2}=\left[\begin{array}{c}
3 \\
1 \\
-1
\end{array}\right], E_{1}=\left[\begin{array}{c}
0.5 \\
1 \\
1
\end{array}\right], F_{1}=\left[\begin{array}{ll}
0 & 1 \\
0 & 0 \\
0 & 1
\end{array}\right], \\
F_{2}=\left[\begin{array}{ll}
0 & 1 \\
0 & 1 \\
0 & 0
\end{array}\right], E_{2}=\left[\begin{array}{cc}
0 & 1 \\
0 & 0.3 \\
0 & 0.5
\end{array}\right],
\end{gathered}
$$

and

$$
C=\left[\begin{array}{lll}
1 & 1 & 1 \\
1 & 0 & 1
\end{array}\right], G=\left[\begin{array}{c}
0.5 \\
1
\end{array}\right], R=\left[\begin{array}{ll}
1 & 0 \\
0 & 0
\end{array}\right]
$$

The weighting functions $\mu_{i}$ are defined as follows

$$
\left\{\begin{array}{l}
\mu_{1}(u(t))=\frac{1-\tanh ((u(t)-1) / 10)}{2} \\
\mu_{2}(u(t))=1-\mu_{1}(u(t))^{2}
\end{array}\right.
$$

The unknown input vector $d(t)$ affects the outputs of the system and its dynamic. The first component of the vector $f(t)$ is a sensor fault and the second component is an actuator fault. $W_{f}$ is chosen to be a diagonal of first order low-pass filters. For each fault, a dedicated residual generator is designed as mentionned above. The resolution of the problem in theorem 1 with $a=0.9$ results in $\gamma_{d}=2.1426$ and $\gamma_{f}=0.5481$ for the first residual generator and for the second residual generator, we choose $a=0.99, \epsilon=\left[\begin{array}{ll}0 & 0.08\end{array}\right]^{T}$ and $b=10$. The solution of the problem in theorem 1 results in $\gamma_{d}=8.2658$ and $\gamma_{f}=1.5780$. The obtained residuals are displayed on figure 2 . The residual $r_{1}(t)$ is sensitive only to the first component of the fault vector (sensor fault) and the second residual $r_{2}(t)$ is only sensitive to the second fault (actuator fault). The filter $W_{f}$ allows to amplify the sensitivity of the residuals to the faults.

A second simulation is performed in order to estimate the faults. $W_{f}$ is then chosen an identity matrix. The original and estimated faults are depicted in figure 3.
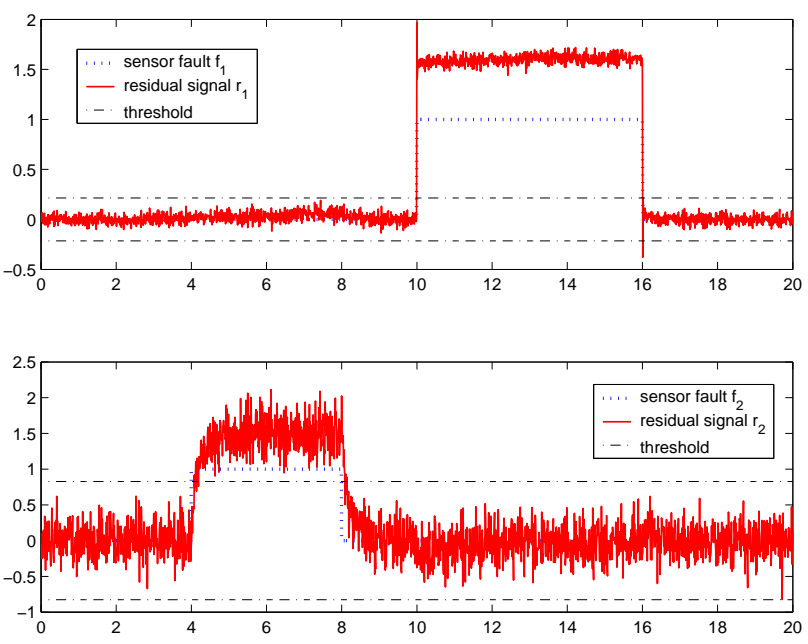

Fig. 2. Faults and corresponding residual signals 

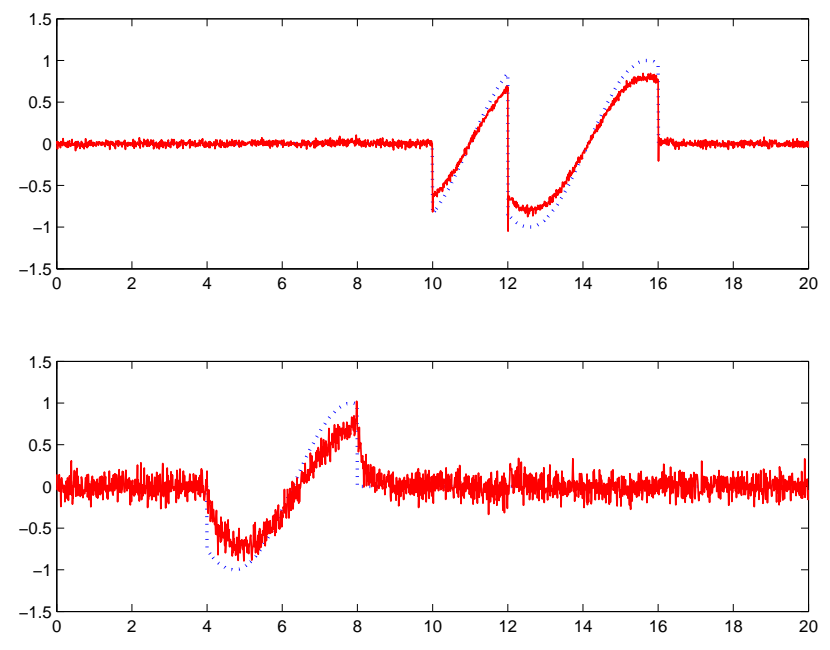

Fig. 3. Faults (dashed lines) and their estimations (solid lines)

\section{CONCLUSION}

Considering nonlinear systems represented by TS systems, two methods for observer-based residual generator (RG) design are proposed. One is devoted to the systems where the premise variables depend on the measured variables such as the input or the output of the system. The other one concerns the systems which premise variables depend on the unmeasured state variables. Sufficient conditions for the existence of RG were established in the LMI formalism in order to ease RG design and obtain the gains. The conservatism of the conditions proposed in the theorem 2 in the case where the premise variable is the state of the system will be studied in future works in order to propose less conservative conditions for residual generators design by using, for example, a non-quadratic Lyapunov function.

\section{REFERENCES}

A. Akhenak, M. Chadli, J. Ragot, and D. Maquin. Design of sliding mode unknown input observer for uncertain TakagiSugeno model. In 15th Mediterranean Conference on Control and Automation, MED'07, Athens, Greece, 2007.

P. Bergsten, R. Palm, and D. Driankov. Observers for TakagiSugeno fuzzy systems. IEEE Transactions on Systems, Man, and Cybernetics - Part B: Cybernetics, 32(1):114-121, 2002.

S. Boyd, L. El Ghaoui, E. Feron, and V. Balakrishnan. Linear Matrix Inequalities in System and Control Theory. SIAM ed., 1994.

A. Casavola, F. Domenico, and G. Franze. Robust fault detection of uncertain linear systems via quasi-lmis. Automatica, 44(1):289-295, January 2008.

M. Chadli, D. Maquin, and J. Ragot. Non quadratic stability analysis of Takagi-Sugeno systems. In IEEE Conference on Decision and Control, CDC'2002, Las Vegas, Nevada, USA, 2002.

J. Chen and H. Zhang. Robust detection of faulty actuators via unknown input observers. International Journal of Systems Science, 22(10):1829-1839, 1991.

J. Chen, R.J. Patton, and H.Y. Zhang. Design of unknown input observers and robust fault detection filters. International Journal of Control, 63(1):85 -105, January 1996.

X. Ding and P.M. Frank. Fault detection via optimally robust detection filters. In 28th IEEE Conference on Decision and Control, 1989.
P. M. Frank and X. Ding. Survey of robust residual generation and evaluation methods in observer-based fault detection systems. Journal of Process Control, 7(6):403-424, December 1997.

T.M. Guerra, A. Kruszewski, L. Vermeiren, and H. Tirmant. Conditions of output stabilization for nonlinear models in the Takagi-Sugeno's form. Fuzzy Sets and Systems, 157(9): 1248-1259, May 2006.

B. Marx, D. Koenig, and D. Georges. Robust fault diagnosis for descriptor systems-a coprime factorization approach. In Proceedings of the 6th IFAC Symposium on Fault Detection, Supervision and Safety of Technical Processes, pages 507$512,2003$.

B. Marx, D. Koenig, and J. Ragot. Design of observers for takagi sugeno descriptor systems with unknown inputs and application to fault diagnosis. IET Control Theory and Application, 1:1487-1495, 2007.

E. Mazars, Z. Li, and I.M. Jaimoukha. A QMI approach to the robust fault detection and isolation problem. In Proceedings of the 6th IFAC Symposium on Fault Detection, Supervision and Safety of Technical Processes, Beijing, China, 2006.

E. Mazars, I. Jaimoukha, and L. Zhenhai. Computation of a reference model for robust fault detection and isolation residual generation. Journal of Control Science and Engineering, 2008. doi:10.1155/2008/790893.

R. Palm and P. Bergsten. Sliding mode observers for TakagiSugeno fuzzy systems. 9th IEEE International Conference on Fuzzy Systems, FUZZ IEEE 2000, San Antonio, TX, USA, 2000.

R. Patton, P. Frank, and R Clark. Fault diagnosis in dynamic systems: Theory and application. Prentice Hall international, 1989.

R.J. Patton, J. Chen, and C.J. Lopez-Toribio. Fuzzy observers for non-linear dynamic systems fault diagnosis. In 37th IEEE Conference on Decision and Control, Tampa, Florida USA, 1998.

J. Stoustrup and H. Niemann. Application of an $\mathrm{H}_{\infty}$ based FDI and control scheme for the three tank system. In Proceedings of the 4th IFAC Symposium on Fault Detection, Supervision and Safety for Technical Processes, Budapest, Hungary, June 2000.

T. Takagi and M. Sugeno. Fuzzy identification of systems and its applications to modeling and control. IEEE Transactions on Systems, Man, and Cybernetics, 15:116-132, 1985.

K. Tanaka and H.O. Wang. Fuzzy Control Systems Design and Analysis: A Linear Matrix Inequality Approach. John Wiley and Sons, inc, 2001.

K. Tanaka, T. Ikeda, and H.O. Wang. Fuzzy regulators and fuzzy observers: Relaxed stability conditions and LMI-based designs. IEEE Transactions on Fuzzy Systems, 6(2):250$265,1998$. 